\title{
Serum $\beta$-klotho is a potential biomarker in the prediction of clinical outcomes among patients with NSCLC
}

\author{
Juan Zhou ${ }^{1,2 \#}$, Suqin Ben ${ }^{3 \#}$, Tan $\mathrm{Xu}^{4 \#}$, Liqin $\mathrm{Xu}^{2}, \mathrm{Xin}^{\mathrm{Yao}}{ }^{1}$ \\ ${ }^{1}$ Department of Respiratory and Critical Care Medicine, The First Affiliated Hospital of Nanjing Medical University, Nanjing, China; ${ }^{2}$ Department \\ of Respiratory and Critical Care Medicine, Affiliated Hospital of Nantong University, Nantong, China; ${ }^{3}$ Department of Respiratory and Critical \\ Care Medicine, Shanghai General Hospital, Shanghai Jiao Tong University, Shanghai, China; ${ }^{4}$ Department of Neurology, Affiliated Hospital of \\ Nantong University, Nantong, China \\ Contributions: (I) Conception and design: X Yao; (II) Administrative support: X Yao; (III) Provision of study materials or patients: J Zhou, L Xu; (IV) \\ Collection and assembly of data: J Zhou, S Ben; (V) Data analysis and interpretation: J Zhou, T Xu; (VI) Manuscript writing: All authors; (VII) Final \\ approval of manuscript: All authors. \\ \#These authors contributed equally to this work. \\ Correspondence to: Xin Yao. Department of Respiratory and Critical Care Medicine, The First Affiliated Hospital of Nanjing Medical University, 300 \\ Guangzhou Road, Nanjing 210029, China. Email: yaoxin@njmu.edu.cn.
}

Background: We aimed to investigate the $\beta$-klotho (KLB) expression in non-small cell lung cancer (NSCLC) and to determine its value as a novel molecular target for survival prognosis in patients with NSCLC.

Methods: The serum KLB concentrations in 50 patients with NSCLC and the 20 healthy persons were measured by enzyme-linked immunosorbent assay (ELISA) methods. The relationship between serum KLB level, including the level change after therapy, and the progression-free survival (PFS) and overall survival (OS) were analyzed. The KLB expression in A549 cells was measured by real-time polymerase chain reaction (RTPCR) and western blotting. The function of cells was revealed by in vitro studies.

Results: The concentrations of serum KLB in patients with NSCLC were obviously lower than those in healthy subjects. KLB expression was significantly increased in patients after chemotherapy and epidermal growth factor receptor tyrosine kinase inhibitor (EGFR-TKI) targeted therapy. In addition, expression of KLB was positively related with PFS and OS. Compared with 16-human bronchial epithelial (HBE) cells, the expression level of KLB was significantly decreased in A549 cells. Overexpression of KLB suppressed the proliferation of A549 cells, along with G1-to-S phase arrest and apoptosis induction.

Conclusions: KLB plays an anti-tumorigenic role in NSCLC. KLB may be a candidate target for the diagnosis and treatment of NSCLC and may serve a potentially significant role in future clinical applications.

Keywords: Non-small cell lung cancer (NSCLC); $\beta$-klotho (KLB); survival prognosis; progression-free survival (PFS); overall survival (OS)

Submitted Apr 08, 2021. Accepted for publication May 18, 2021.

doi: $10.21037 /$ jtd-21-798

View this article at: http://dx.doi.org/10.21037/jtd-21-798

\footnotetext{
^ ORCID: 0000-0001-5803-0948.
} 


\section{Introduction}

Lung cancer is the most common cancer, and leads to about $25 \%$ of cancer deaths. Of these lung cancer-related deaths, non-small cell lung cancer (NSCLC) accounts for about $85 \%$. Despite there being numerous methods used for the earlier diagnosis of NSCLC, such as low-dose spiral computed tomography (CT), the 5-year survival rate of this disease is still less than $20 \%$ after administration of antitumor therapies, including chemotherapy, targeted therapy, and even immunotherapy.

One major reason for this unsatisfactory outcome is that most lung cancer cases are diagnosed at an advanced stage, as the means for a more effective early diagnosis is lacking (1).

Klotho was originally recognized to be a powerful ageing-suppressor gene (2). In animal studies, Klotho deficiency has been observed to result in the onset of numerous aging phenotypes, including severe growth retardation, premature organ degeneration, arteriosclerosis, and a subsequently shortened lifespan (3). In contrast, overexpression of Klotho in mice using viral delivery or genetic manipulation has been shown to extend life by $20-30 \%(4-6)$.

The Klotho family consists of three members, $\alpha$-klotho (KLA), $\beta$-klotho (KLB), and $\gamma$-klotho. In addition to being closely associated with ageing-related diseases, such as chronic obstructive pulmonary disease and idiopathic pulmonary fibrosis, Klotho has been also shown to be related with the prognosis of variety types of cancers (7-10), with its expression potentially decreasing in cancer due to histone deacetylation and hypermethylation in the promoter region. This phenomenon has been observed in many types of tumors, including those of hepatocellular carcinoma (11), gastric carcinoma (12), and esophageal squamous cell carcinoma (13).

As one of the Klotho family members, KLB is a singlepass transmembrane protein composed of 1,043 amino acids. It was found to mainly express in liver $(14,15)$, pancreas and adipose tissues, and has been shown to have more complex role in studies of various cancers. Ye and Lee $(16,17)$ demonstrated KLB level to be significantly reduced in liver tissue fibrosis and to suppress tumor growth in hepatocellular carcinoma. Meanwhile, Liu (18) reported that KLB promoted the inhibitory function of metformin in endometrial adenocarcinoma, while it was further discovered that KLB suppresses the capacity of migration and invasion in cervical cancer cells (19). KLB has been studied to identify as a FGFR4 co-receptor, which is required for high affinity binding and activity of FGF19 in specific liver functions (20). FGF19 forms the FGF19-FGFR4KLB complex to activate multiple downstream signaling pathways to exert its function dependent of KLB (21). Motylewska et al. (22) reported for the first time that KLB level was decreased in the thyroid cancer, but with the elevation of two other components of the FGF19/FGFR4/ KLB signaling pathway in these patients. The finding indicated that KLB may through dysfunction of FGF19/ FGFR4/KLB signaling pathway playing a role in thyroid cancer development. In contrast, Poh et al. (23) reported that KLB plays a tumorigenic role in hepatocellular carcinoma. Thus, KLB may be tumor suppressive or tumorigenic, depending on the cancer type. Regardless, the degree to which KLB plays a role in NSCLC has not been extensively investigated.

In this study, we observed the expression levels of KLB in the serum of NSCLC patients and A549, explored its clinical association with progression-free survival (PFS) and overall survival (OS), and evaluated its effects on cell function. Univariate and multivariate analyses were performed to evaluate prognostic values of this biomarker for patient survival.

Based on our findings, we posit that KLB may serve as a potential diagnostic biomarker for NSCLC.

We present the following article in accordance with the REMARK reporting checklist (available at http://dx.doi. org/10.21037/jtd-21-798).

\section{Methods}

\section{Clinical data}

A total of 50 NSCLC patients were pathologically or cytologically confirmed as IIIb or IV stages according to the eighth American Joint Committee on Cancer staging system and the Union for International Cancer Control tumor-node-metastasis (TNM) classification system. A total of 70 serum samples, including 50 from NSCLC patients and 20 from healthy individuals, were taken from the Affiliated Hospital of Nantong University between January 2019 and June 2020 prospectively. The 20 healthy individuals were aged $40-75$ years and were recruited from the physical examination center, and were screened to exclude hypertension, diabetes, kidney disease, coronary heart disease, and other diseases. All procedures performed in this study involving human participants were in accordance with the Declaration of Helsinki (as revised 
Table 1 The specific sequences of primers for RT-PCR

\begin{tabular}{ll}
\hline Primer & Sequence (5' to 3') \\
\hline Human GAPDH & Forward primer: 5'-GGAGCTGACTTCGGAACTAAAGG-3' \\
& Reverse primer: 5'-TGTGGTTTCAGGGAGGCC-3' \\
Human KLB & Forward primer: 5'-GCCATCATCGCACAAGAATCC-3' \\
& Reverse primer: 5'-CTTACCTGTTTGCTCCTTTCAAGAG-3' \\
\hline
\end{tabular}

RT-PCR, real-time polymerase chain reaction; KLB, $\beta$-klotho.

in 2013). All participants gave informed consent and agreed to participate in the study. The study was approved by the Institute Research Ethics Committee of Affiliated Hospital of Nantong University (No. 2018-L100).

\section{Treatment evaluations}

Evaluation of the efficacy of treatment began at two cycles for chemotherapy or at 2 months after the initiation of drug administration for epidermal growth factor receptor tyrosine kinase inhibitors (EGFR-TKIs). Complete response (CR), partial response (PR), stable disease (SD), and progressive disease (PD), PFS, and OS were used for efficacy evaluation and as survival analysis indicators.

\section{Serum KLB measurement}

The serum specimens were obtained from patients before and after treatment (two cycles for chemotherapy or 2 months of EGFR-TKI administration). After at least $8-10$ hours of fasting, blood samples were collected within 24 hours. All serum samples were immediately frozen at $-80{ }^{\circ} \mathrm{C}$ before laboratory testing. KLB levels were measured with a commercially available enzyme-linked immunosorbent assay (ELISA) kit (R\&D Systems China, DY5889-05), according to the manufacturer's protocol.

\section{Cell culture}

A549 cells and 16-human bronchial epithelial (HBE) cells (ScienCell Research Laboratories, Carlsbad, CA, USA) were cultured at $37{ }^{\circ} \mathrm{C}$ in a $95 \%$ air and $5 \% \mathrm{CO}_{2}$ incubator in RPMI 1640 medium supplemented with $10 \%$ fetal bovine serum (FBS) and $1 \%$ penicillin/streptomycin.

\section{KLB plasmid overexpression}

A549 were plated in $10 \%$ FBS without antibiotics. The human KLB overexpression plasmid pcDNA3.1-3xflag-c (KLB-OE) was purchased from Talen-bio (Wuxi, China), and empty plasmid (KLB-EV) was used as the control. According to the manufacturer's instructions, the plasmids were transfected into cells using Lipofectamine ${ }^{\mathrm{TM}} 2000$ (Invitrogen, USA) the next day to produce cells which had a higher KLB expression. The expression of KLB protein was measured by western blotting after 72 hours.

\section{$R N A$ extraction and quantitative real-time polymerase chain reaction ( $q R T-P C R)$}

RNA was isolated from cultured cells using Trizol reagent (Invitrogen, Carlsbad, CA, USA) according to the manufacturer's protocol. Total RNA $(1 \mu \mathrm{g})$ was used as a template for reverse transcription to complement DNA (cDNA) with PrimeScript Reverse Transcriptase (Takara, Kyoto, Japan). qRT-PCR was carried out by using SYBR qRCR premix (Takara, Kyoto, Japan). The primers (Invitrogen) used are presented in Table 1. The cycling parameters were as follows: predenaturation at $94{ }^{\circ} \mathrm{C}$ for 5 minutes, 30 cycles of denaturation at $94^{\circ} \mathrm{C}$ for 30 seconds, annealing at $60{ }^{\circ} \mathrm{C}$ for 30 seconds, extension at $72{ }^{\circ} \mathrm{C}$ for 30 seconds, and a final extension at $72{ }^{\circ} \mathrm{C}$ for 10 minutes.

\section{Western blotting}

Total proteins were extracted from cells by using RIPA lysis buffer containing protease inhibitors (Beyotime, China). Then, $20 \mathrm{~g}$ of protein was loaded onto $10 \%$ SDS-PAGE and transferred to polyvinylidene fluoride (PVDF) membranes (Millipore, Bedford, MA, USA). Anti-KLB antibodies (1:1,000; AF5889; R\&D) and anti-GAPDH (1:1,000; 5174; Cell Signaling Technology, Danvers, MA, USA) were used as the primary antibodies. Incubation was conducted with the secondary horseradish peroxidase (HRP)-linked antiRabbit immunoglobin G (IgG) antibody (1:5,000; Bioworld, Shanghai, China) for 1 hour at $20^{\circ} \mathrm{C}$. After being rinsed 
with Tris-buffered saline with $0.1 \%$ Tween 20 , the blots were detected by an enhanced chemiluminescence (ECL) kit plus reagent (ECL, Thermo Fisher Scientific, Waltham, USA). GAPDH was used as the housekeeping gene for normalization.

\section{Cell proliferation analysis}

Cell counting kit-8 (CCK-8 kit; Tongren, Shanghai, China) was used to assess cell viability. The KLB-EV and KLBOE A549 cells were seeded on 96-well plates at a density of $5 \times 10^{3}$ cells/well. The medium without cells containing CCK8 was the blank control. Each well was incubated with $10 \mu \mathrm{L}$ of CCK-8 reagent for 2 hours at $37^{\circ} \mathrm{C}$. Absorbance was then measured at $450 \mathrm{~nm}$ with a microplate reader (Thermo Fisher Scientific).

\section{Cell cycle analysis}

A single-cell suspension of $2 \times 10^{6}$ cells $/ \mathrm{ml}$ placed in ice-cold phosphate-buffered saline (PBS) was prepared from both KLB-EV and KLB-OE A549 cells, and then 70\% ethanol were used for fixing at $4{ }^{\circ} \mathrm{C}$ for 12 hours. Subsequently, $500 \mu \mathrm{L}$ PI/Triton X-100 solution was added into the cell suspensions for 15 minutes at $37^{\circ} \mathrm{C}$. Cell clumps were removed through a cell strainer (BD BioSciences, CA, USA) and the number of stained cells were assessed by BD Accuri Cytometry (BD BioSciences, Franklin Lakes, NJ, USA).

\section{Flow cytometry-based Annexin V/PI assay}

The levels of cells apoptosis were measured by Annexin V/ PI double-staining in both KLB-EV and KLB-OE A549 cells. The cells were digested with $0.25 \%$ trypsin-EDTA and resuspended to a concentration of $1 \times 10^{6}$ cells $/ \mathrm{mL}$ in PBS. Next, $10 \mu \mathrm{L}$ of Annexin V-FITC was added into suspended cells at room temperature for 15 minutes in the dark, after which $5 \mu \mathrm{L}$ of propidium iodide solution was added into the cell suspensions. Cell analysis was measured by BD Accuri Cytometry (BD Biosciences).

\section{Statistical analysis}

We divided participants into different groups according to the median value of baseline KLB levels, posttherapy KLB levels and difference KLB values in pre- and posttreatment, and evaluated the difference between pre- and posttreatment levels. Baseline information was compared using $\chi^{2}$ tests.
Cumulative rates of progression and death were calculated for different groups using the Kaplan-Meier method and were compared using the log-rank test. Unadjusted and adjusted hazards ratios (HRs) with $95 \%$ confidence intervals (CIs) were used for the association of baseline KLB levels, posttherapy KLB levels, their differences, and other confounding factors for progression and death were assessed by univariate and multivariate Cox proportional hazards models. Statistical analysis was conducted using GraphPad Prism software v.5.0 (GraphPad Software, Inc., San Diego, CA, USA). All statistical tests were two-tailed, and a $\mathrm{P}$ value $<0.05$ was considered statistically significant.

\section{Results}

\section{Serum KLB levels were decreased in NSCLC}

The serum levels of KLB in lung cancer patients and healthy individuals were analyzed. KLB serum levels in NSCLC patients were $324(278.25-380) \mathrm{ng} / \mathrm{\mu L}$, which was significantly lower than the $707.5(689.75-805.25) \mathrm{ng} / \mathrm{\mu L}$ found in the control group.

\section{Correlation of serum KLB expression with clinicopathologic features by chi-square test}

The median level of KLB, including baseline KLB, posttherapy KLB, and difference KLB values in pre- and posttreatment, was set as a cutoff point to divide cases into two groups: a high-level group and a low-level group (Tables 2-4). The correlation of serum KLB expression with clinicopathologic features was determined by chi-square test.

Those cases with EGFR mutations and treated with EGFR-TKIs had a high baseline serum KLB level (KLB $>324 \mathrm{pg} / \mathrm{mL}$; Table 2). A higher correlation was also observed between patients with EGFR mutations treated with EGFR-TKI and those with a high posttherapy KLB level (KLB >391 pg/mL; Table 3). In addition, Patient with high posttherapy KLB levels tended to be the male and the younger (<65 years) (Table 3).

The median level of KLB difference was $55 \mathrm{pg} / \mathrm{mL}$ (Table 4). A significant increase in KLB difference level was found in male patients after treatment (Table 4); however, age, pathology, TNM stage, smoking status, Eastern Cooperative Oncology Group performance status (ECOG PS), treatment, and EFGR mutation type were not significantly associated with a difference in KLB level.

The pathologies of adenocarcinoma and squamous types 
Table 2 Correlation of baseline KLB expression with clinicopathologic features

\begin{tabular}{|c|c|c|c|}
\hline Variables & Baseline KBL $\leq 324 \mathrm{pg} / \mathrm{mL}$ & Baseline KLB > 324 pg/mL & $P$ value \\
\hline Gender, n (\%) & & & 0.39 \\
\hline Male & $13(52.0)$ & $16(64.0)$ & \\
\hline Female & $12(48.0)$ & $9(36.0)$ & \\
\hline$<65$ & $16(64.0)$ & $14(56.0)$ & \\
\hline$\geq 65$ & $9(36.0)$ & $11(44.0)$ & \\
\hline Pathology, n (\%) & & & 0.16 \\
\hline Adenocarcinoma & $18(72.0)$ & $22(88.0)$ & \\
\hline Illb & $7(28.0)$ & $5(20.0)$ & \\
\hline IV & $18(72.0)$ & $20(80.0)$ & \\
\hline Smoking, n (\%) & & & 0.57 \\
\hline Nonsmoker & $12(48.0)$ & $14(56.0)$ & \\
\hline Smoker & $13(52.0)$ & $11(44.0)$ & \\
\hline ECOG PS, n (\%) & & & 0.73 \\
\hline 0 & $22(88.0)$ & $20(80.0)$ & \\
\hline 1 & $2(8.0)$ & $3(12.0)$ & \\
\hline EFGR type, n (\%) & & & $<0.0001$ \\
\hline Unmeasured & $9(36.0)$ & $3(12.0)$ & \\
\hline Wild-type & $12(48.0)$ & $3(12.0)$ & \\
\hline Mutation & $4(16.0)$ & $19(76.0)$ & \\
\hline
\end{tabular}

KLB, $\beta$-klotho; TNM, tumor-node-metastasis; ECOG PS, Eastern Cooperative Oncology Group performance status; EGFR-TKI, epidermal growth factor receptor tyrosine kinase inhibitor.

were found to not be significantly associated with any of baseline $\mathrm{KLB}$, posttherapy KLB, and difference KLB levels (Tables 2-4).

\section{Characterization of serum $K L B$ expression and its relevance with survival prognosis of NSCLC}

To assess the value of KLB for prognosis in NSCLC, we analyzed the relationship between serum KLB expression and survival outcomes. The median PFS and OS of the 50 patients were 9 (range, 6-13) and 16.5 (range, 12-20) months, respectively. As shown in Table 5, higher KLB levels at baseline, posttherapy, and their difference were significantly and negatively associated with shorter PFS and OS.

Moreover, those patients with adenocarcinoma, EGFR mutations and treated with EGFR-TKIs had better survival outcomes. 
Table 3 Correlation of posttherapy KLB expression with clinicopathologic features

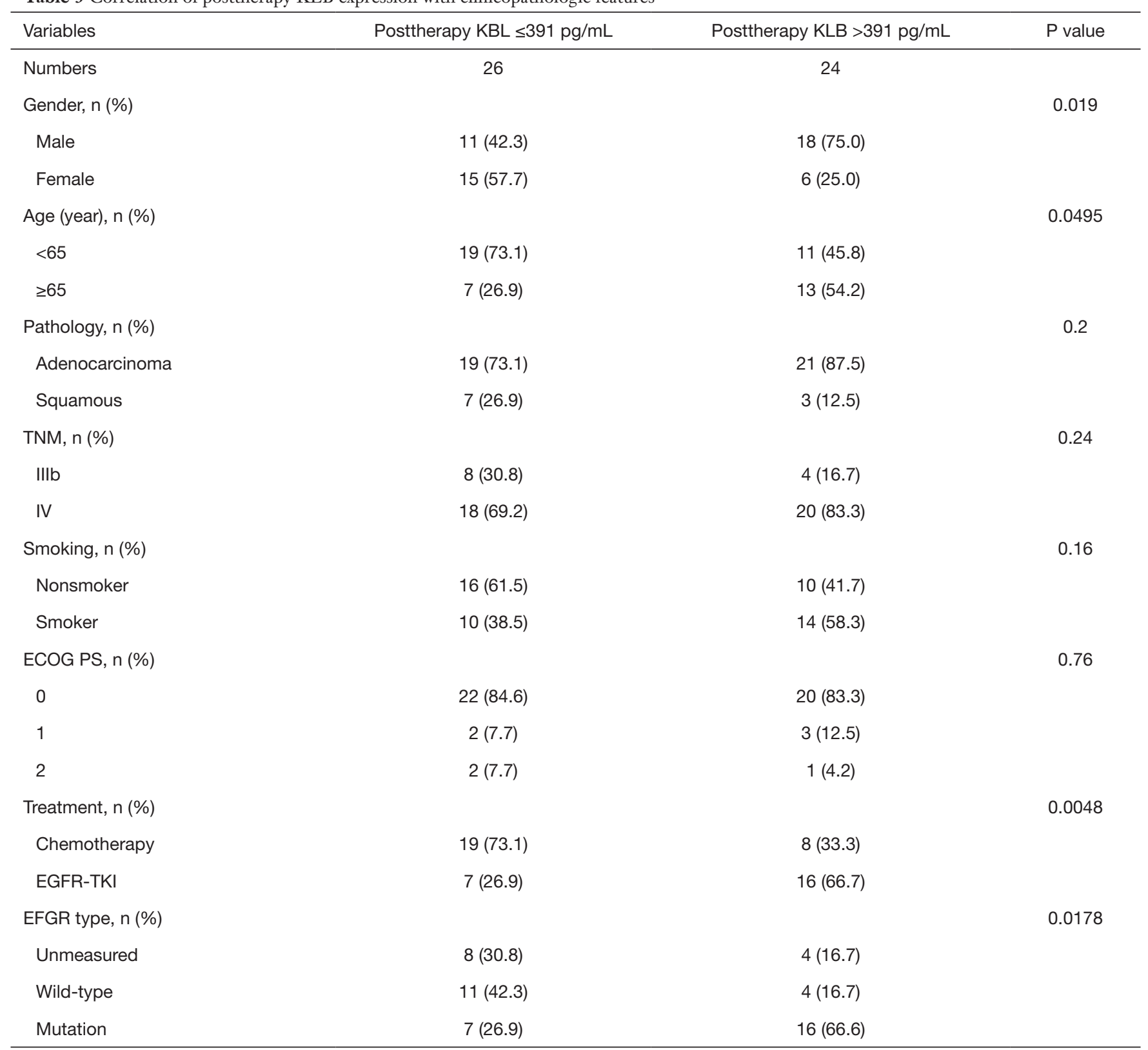

KLB, $\beta$-klotho; TNM, tumor-node-metastasis; ECOG PS, Eastern Cooperative Oncology Group performance status; EGFR-TKI, epidermal growth factor receptor tyrosine kinase inhibitor.

Kaplan-Meier analysis of the association of serum KLB expression level with PFS and OS in NSCLC

Results of the Kaplan-Meier analysis of serum expression level of KLB in relation to the PFS and OS of NSCLC patients is shown in Figure 1.

We found that higher KLB levels at baseline, post therapy, and a larger differential value correlated with longer PFS and OS compared with lower KLB levels in NSCLC.

\section{Multivariate analysis of prognostic factors with PFS and OS}

The prognostic capacity of serum KLB expression on survival was evaluated with multivariate analysis. As summarized in Table 6, the multivariate analysis revealed 
Table 4 Correlation of difference in KLB expression with clinicopathologic features

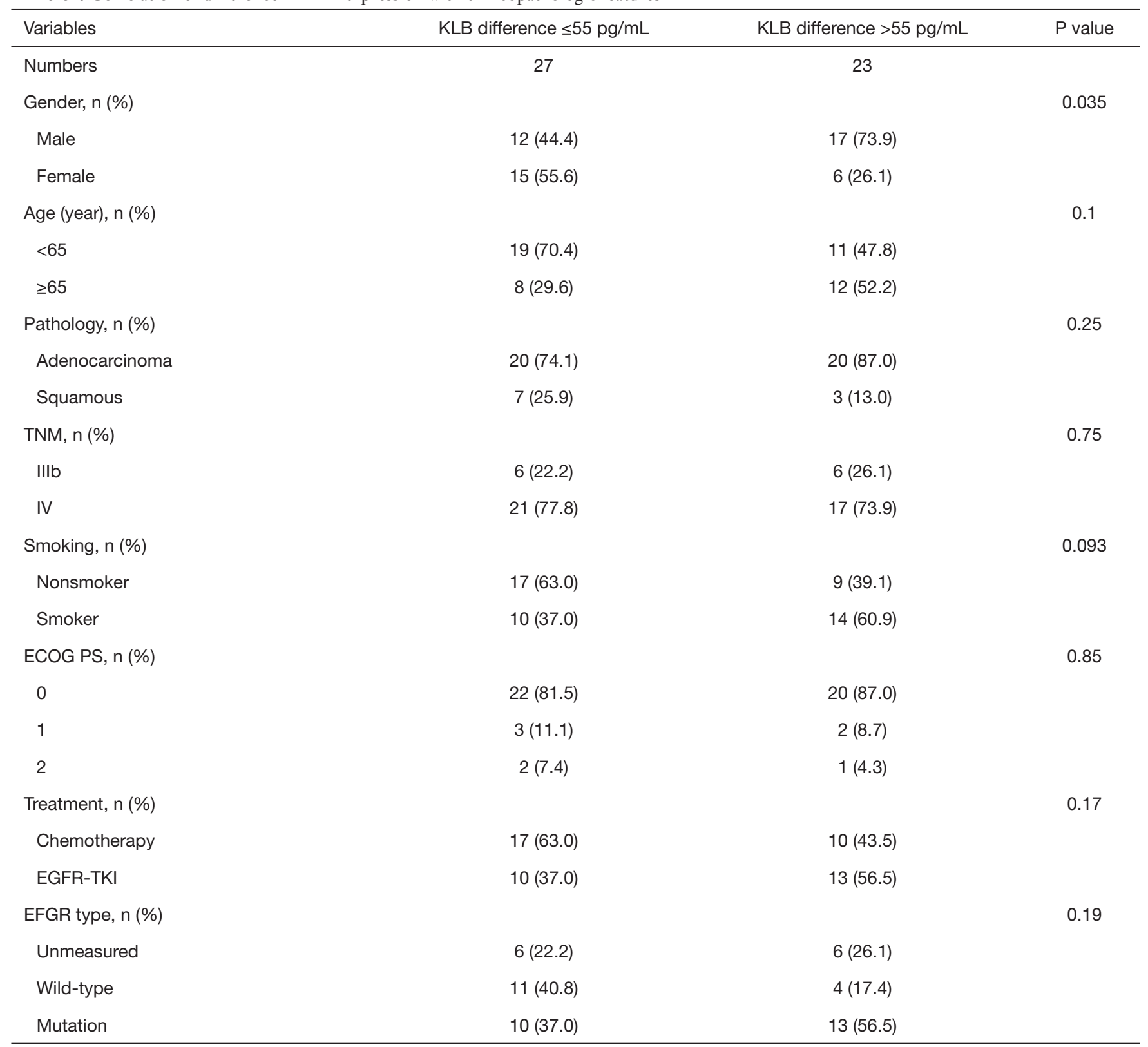

KLB, $\beta$-klotho; TNM, tumor-node-metastasis; ECOG PS, Eastern Cooperative Oncology Group performance status; EGFR-TKI, epidermal growth factor receptor tyrosine kinase inhibitor.

that baseline and posttherapy KLB expression levels were independent prognostic factors for both PFS and OS. Those patients with a higher differential value of KLB before and after treatment exhibited a significantly longer PFS, but this showed no association with OS.
KLB was decreased in A549 cells compared with $16 \mathrm{HBE}$ cells, and overexpression KLB by plasmid transfection led to increased KLB expression in A549 cells

KLB expression in NSCLC cells was assessed. Compared with $16 \mathrm{HBE}$ cells, expression levels of KLB were decreased 
Table 5 Univariate analyses of factors associated with survival outcomes

\begin{tabular}{lcccccc}
\hline \multirow{2}{*}{ Variables } & \multicolumn{2}{c}{ PFS } & & \multicolumn{2}{c}{ OS } \\
\cline { 2 - 5 } & P value & HR & $95 \%$ Cl & P value & HR & $95 \%$ Cl \\
\hline Age (>65 vs. $\leq 65)$ & 0.6732 & 0.875 & $0.470-1.629$ & 0.5427 & 0.780 & $0.351-1.734$ \\
Sex (male vs. female) & 0.7429 & 1.109 & $0.598-2.054$ & 0.3962 & 1.419 & $0.632-3.188$ \\
Pathology (adenocarcinoma vs. & 0.0044 & 3.443 & $1.471-8.059$ & 0.0402 & 2.660 & $1.045-6.777$ \\
squamous) & & & & 0.4295 & 1.489 \\
TNM & 0.8318 & 1.081 & $0.5274-2.219$ & $0.555-3.999$ \\
Smoking & 0.2516 & 1.441 & $0.772-2.689$ & 0.4772 & 1.331 \\
ECOG & 0.2618 & 0.702 & $0.379-1.302$ & 0.9954 & 0.998 & $0.499-1.994$ \\
Treatment (TKI vs. chemotherapy) & $<0.0001$ & 0.064 & $0.020-0.201$ & 0.0002 & 0.151 & $0.055-0.410$ \\
EGFR (+) & $<0.0001$ & 0.070 & $0.021-0.238$ & 0.0005 & 0.153 & $0.053-0.439$ \\
KLB baseline & $<0.0001$ & 0.079 & $0.028-0.222$ & $<0.0001$ & 0.160 & $0.064-0.401$ \\
KLB posttherapy & 0.0010 & 0.303 & $0.149-0.617$ & 0.0304 & 0.403 & $0.177-0.917$ \\
KLB difference & $<0.0001$ & 0.070 & $0.021-0.238$ & 0.0005 & 0.153 & $0.053-0.439$ \\
\hline
\end{tabular}

PFS, progression-free survival; OS, overall survival; HR, hazards ratio; Cl, confidence interval; TNM, tumor-node-metastasis; ECOG, Eastern Cooperative Oncology Group; TKI, tyrosine kinase inhibitor; EGFR, epidermal growth factor receptor; KLB, $\beta$-klotho.

in A549 cells (Figure 2A,B).

Since KLB expression was inversely correlated with NSCLC progression, we further examined the function of KLB in NSCLC cells. After KLB-OE was transfected into A549, KLB expression levels were determined by western blotting. The KLB protein level was significantly higher in the KLB-OE group than in the KLB-EV control group (Figure 2C).

\section{Overexpression of KLB induced G1-to-S phase arrest and led to the apoptosis and weakened viability of $A 549$ cells}

The proliferative effect of KLB was then evaluated. Overexpression of KLB in A549 cells was accompanied by an increase in the proportion of cells in G0/G1 stage and a decrease in the proportion of cells in S stage (Figure $3 A$ ). This indicated that the higher expression level of KLB induced G1-to-S phase arrest of NSCLC cells. Flow cytometry assay was then conducted to evaluate whether KLB could induce apoptosis in A549 cells. Overexpression of KLB in A549 cells markedly increased the levels of apoptosis in both the early and late stages (Figure 3B). These results demonstrated that KLB inhibition of cell proliferation was associated with G1-to-S phase arrest and an induction of apoptosis. Moreover, KLB overexpression resulted in a significant inhibition of cell viability (Figure 3C).

\section{Discussion}

In China, the mortality and morbidity of lung cancer ranks top among all cancer types in both males and females (24). The incidence of lung cancer has gradually risen in China with the rapid intensification of industrialization and environmental pollution $(25,26)$.

Therefore, it has become necessary to clarify the molecular mechanism underlying the occurrence of NSCLC and to identify markers for NSCLC and gene therapy. We thus aimed to investigate the functional role of KLB in NSCLC, and assess the value of KLB in acting as a new target for diagnosis and treatment.

In this study, we found that KLB serum levels were significantly lower in NSCLC patients than in healthy controls. Consistent with our clinical findings, decreased KLB expression levels were also detected in the A549 human lung cancer cell line compared with $16 \mathrm{HBE}$ cells in vitro. These data demonstrate that KLB expression level is decreased in NSCLC.

We then analyzed the correlation of baseline serum KLB expression with clinicopathologic features and its prognostic value for NSCLC survival. Higher KLB serum levels were observed in those who had EGFR mutations and who were treated with EGFR-TKIs, while lower levels of baseline KLB were associated with shorter PFS and OS. Multivariate 
A
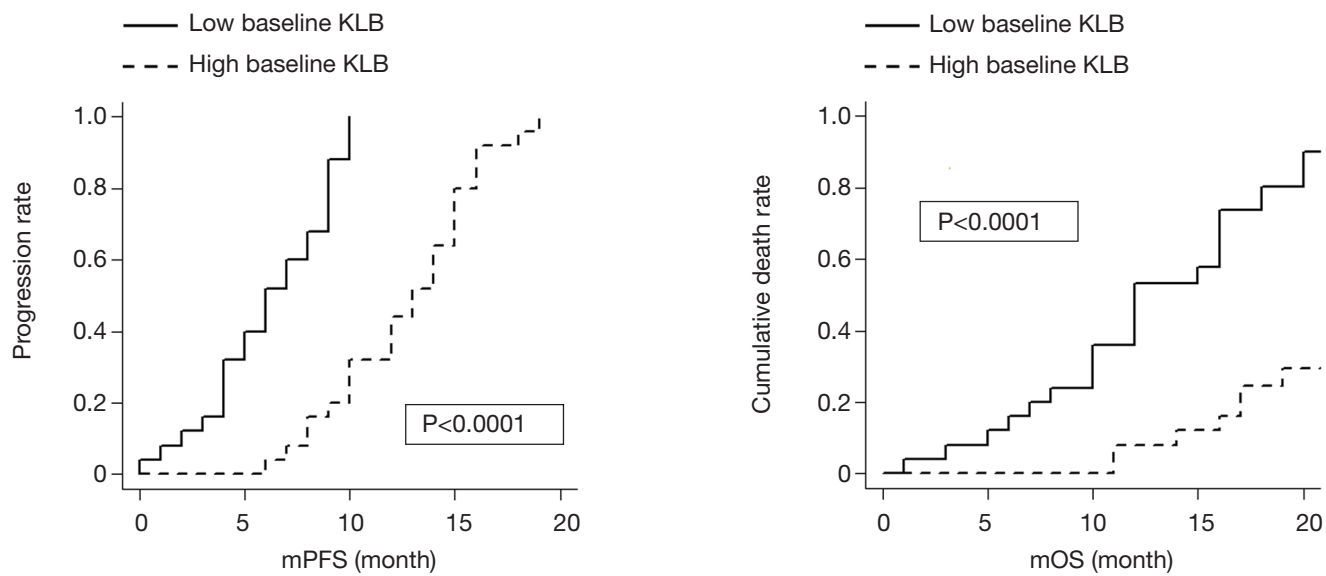

B
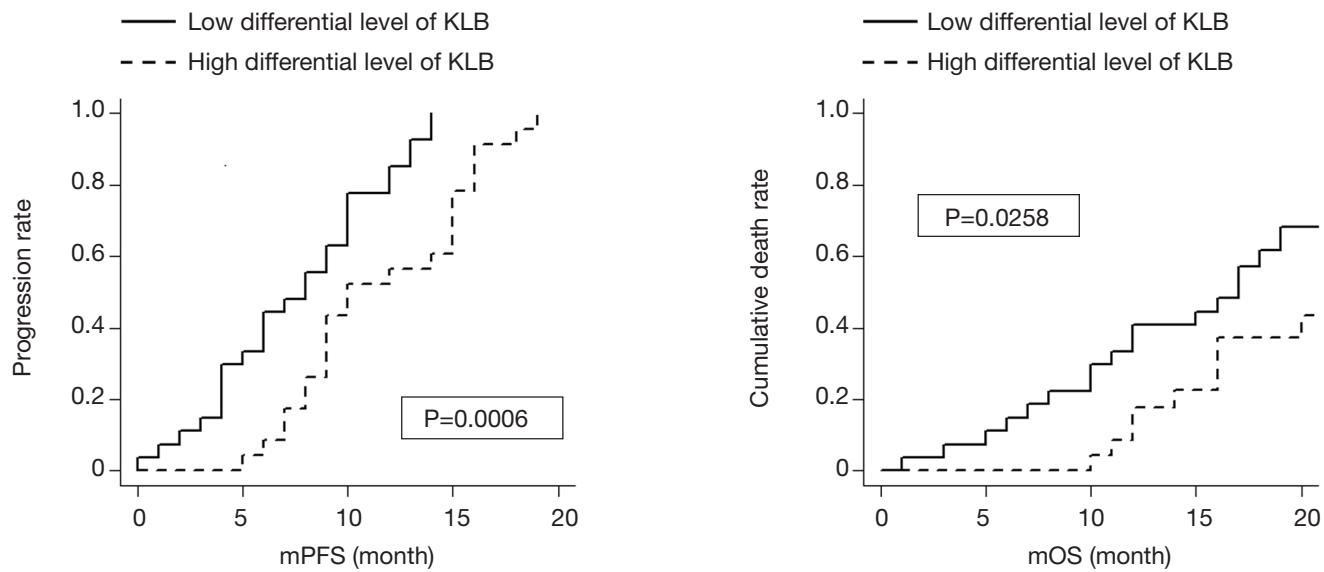

C
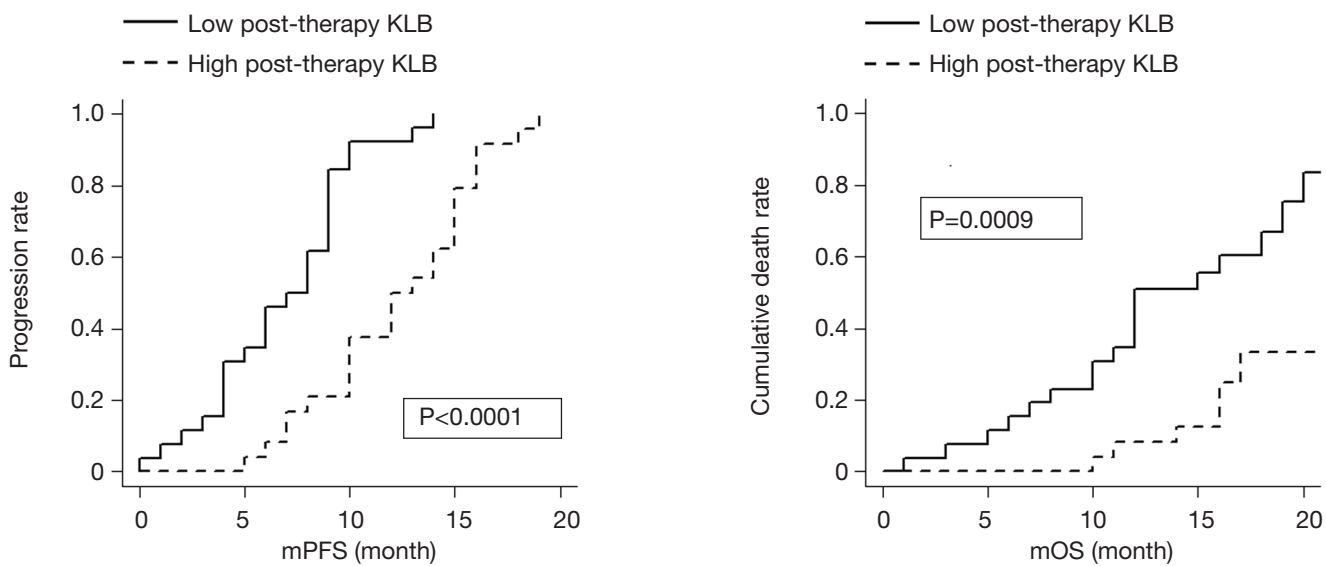

Figure 1 Kaplan-Meier analysis of serum expression level of KLB on PFS and OS in NSCLC patients. (A) KLB level at baseline was associated with PFS and OS; (B) difference in KLB level was correlated with PFS and OS; (C) posttherapy KLB level was associated with PFS and OS. KLB, $\beta$-klotho; PFS, progression-free survival; OS, overall survival; NSCLC, non-small cell lung cancer. 
Table 6 Multivariate analyses of the association between KLB and PFS and OS

\begin{tabular}{|c|c|c|c|c|c|c|}
\hline KLB level & \multicolumn{3}{|c|}{ PFS } & \multicolumn{3}{|c|}{ OS } \\
\hline KLB baseline & 0.0051 & 0.151 & $0.040-0.567$ & 0.0045 & 0.106 & $0.022-0.505$ \\
\hline KLB post therapy & 0.0003 & 0.131 & $0.044-0.390$ & 0.0033 & 0.135 & $0.035-0.514$ \\
\hline KLB difference & 0.0013 & 0.202 & $0.076-0.536$ & 0.1154 & 0.424 & $0.146-1.234$ \\
\hline
\end{tabular}

KLB, $\beta$-klotho; PFS, progression-free survival; OS, overall survival; HR, hazards ratio; Cl, confidence interval.

A

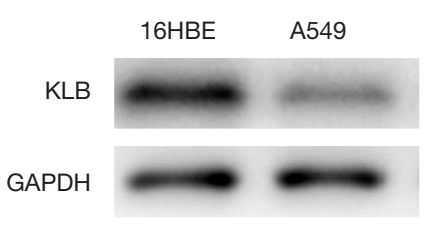

C

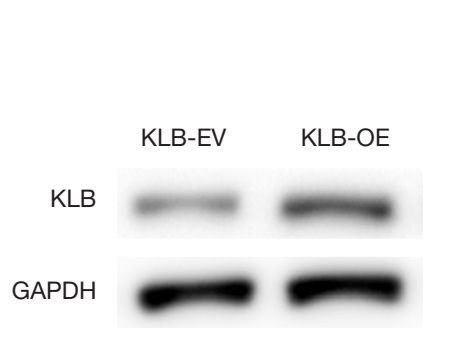

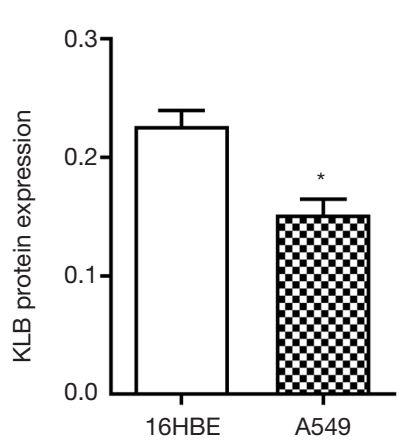

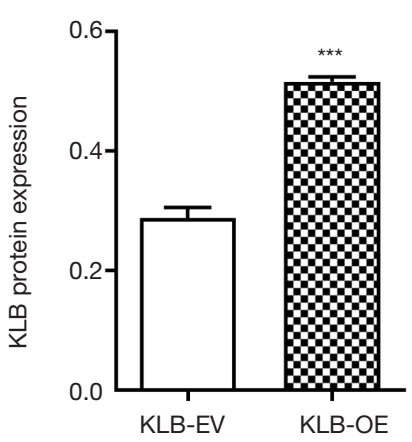

B

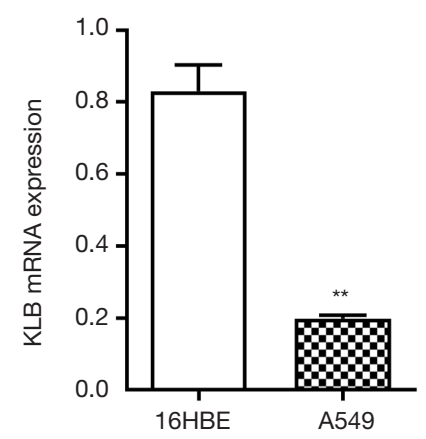

Figure 2 KLB was decreased in A549 cells compared with 16HBE cells, and overexpression of KLB by plasmid transfection led to an increase in KLB expression in A549 cells. (A) KLB protein levels were decreased in A549 compared with 16HBE cells; (B) KLB mRNA expression was decreased in A549 compared with 16HBE cells; (C) transfection with KLB-OE or KLB-EV in A549 cells. Overexpression of KLB protein in A549 cells was revealed by western blotting. * $\mathrm{P}<0.05$; **, $\mathrm{P}<0.01$; *** $\mathrm{P}<0.001$. KLB, $\beta$-klotho; HBE, human bronchial epithelial.

analysis also identified baseline KLB expression as an independent prognostic factor for both PFS and OS. These results are consistent with other recent studies $(27,28)$. Therefore, it is highly likely that KLB contributes to tumor inhibition in NSCLC.

As KLB expression was found to be inversely associated with NSCLC progression, we then investigated the functional effects of KLB on NSCLC cells. Luo found that KLB could induce apoptosis in prostate cancer cells (29).
The overexpression of KLB by plasmid transfection in A549 cells further demonstrated an antitumor effect. Overexpression of KLB induced the G1-to-S phase arrest of NSCLC cells and promoted apoptosis in both the early and late stages, which is in line with the findings of Luo et al. $(29,30)$. Moreover, KLB overexpression led to a significant inhibition of cell viability.

However, no studies have yet examined the effect of cancer treatment on KLB expression level and its relevance 
A
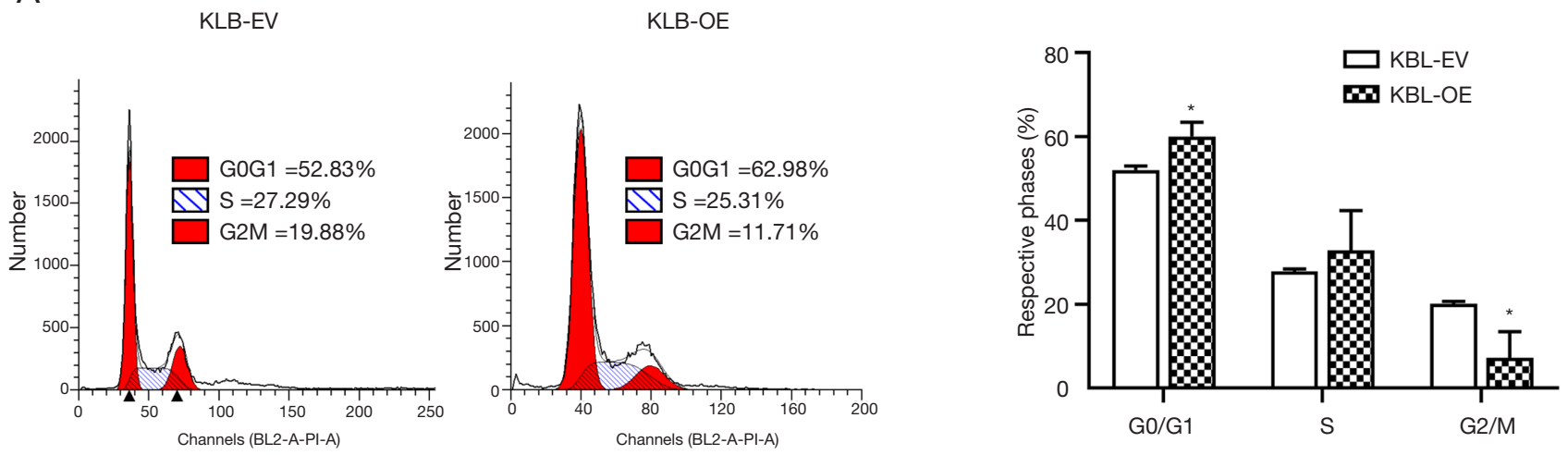

B
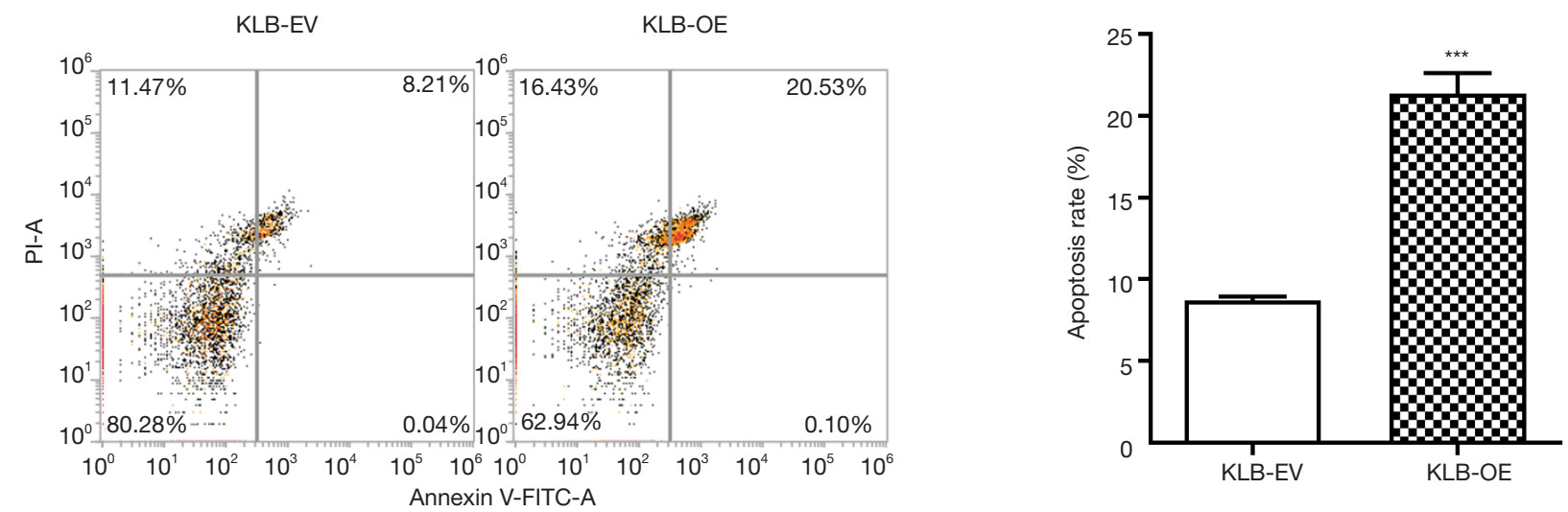

C

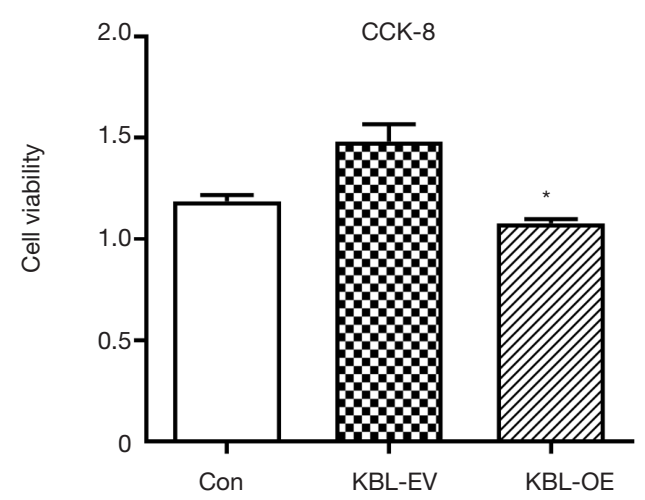

Figure 3 KLB overexpression induced G1-to-S phase arrest, and led to the apoptosis and weakened viability of A549 cells. (A) Representative histograms of cell cycle of A549 transfected with KLB-OE or KLB-EV; (B) in A549, Annexin V/PI assays were performed to assess apoptosis; (C) CCK-8 assay to detect the effect of KLB overexpression on cell viability in A549. Data were collected from three independent experiments. * $\mathrm{P}<0.05$; ${ }^{* * *}, \mathrm{P}<0.001$. KLB, $\beta$-klotho; CCK- 8 , cell counting kit-8. 
to prognosis. We thus further observed the changes in KLB levels after treatment with EGFR-TKI or two cycles of chemotherapy. We showed for the first time that the higher correlation were observed between EGFR mutations, treated with EGFR-TKI and post-treatment KLB level. Univariate analyses indicated that a higher posttreatment KLB level after successful treatment of NSCLC may lead to better survival outcome. The posttherapy KLB level may also be an independent prediction factor for better PFS and OS: the higher the difference between pre- and post-KLB level, the better the survival prognosis. Those patients with a higher differential KLB value exhibited a significantly longer PFS. Therefore, higher posttreatment KLB, and higher differential KLB levels were associated with better NSCLC survival prognosis as well as higher baseline KLB. These findings are in accordance with the view that KLB serves as a tumor suppressor (31).

The mechanism of KLB tumor suppressive effect seems to be complicated. The FGF19/FGFR4/KLB axis presents a key role in the progression of many cancer types. The inhibition of KLB-mediated FGF19 signaling leads to a cell proliferation reduction in colorectal carcinoma through the ERK and $\beta$-catenin pathways inhibition (32). It was also reported that KLB in partnership with FGFR4 inhibited tumor cell proliferation and induced apoptosis in liver cancer, which was related with suppression of the AKT and mTOR pathways (33). Thus, more experiments should be pursued to assess the role of KLB and FGF19/FGFR4 activation in cancer development.

This current study has several limitations. The underlying molecular mechanism of the tumor-suppressing effect of KLB is not well understood. KLB may inhibit cancer via the AKT and mTOR pathways (29), FGFR signaling (34), or via IGF1 signaling pathway (35), but this should be more intensively examined in future research. In the over-expression of KLB, a normal control group should be added. And a KLB knockdown group also needed to observe KLB function. It will be more convincing to test the function of KLB in NSCLC cells by using animal xenograft. We will do more work about it next. Furthermore, whether KLB can serve as an index for survival in NSCLC patients needs to be verified in future studies with a greater sample size.

In conclusion, KLB plays a tumor suppressive role in NSCLC. The KLB expression levels, including those after treatment, may help determine the outcome of patient survival. KLB may potentially serve as a valuable diagnostic and prognostic role in NSCLC care.

\section{Acknowledgments}

Funding: None.

\section{Footnote}

Reporting Checklist: The authors have completed the REMARK reporting checklist. Available at http://dx.doi. org/10.21037/jtd-21-798

Data Sharing Statement: Available at http://dx.doi. org/10.21037/jtd-21-798

Conflicts of Interest: All authors have completed the ICMJE uniform disclosure form (available at http://dx.doi. org/10.21037/jtd-21-798). The authors have no conflicts of interest to declare.

Ethical Statement: The authors are accountable for all aspects of the work in ensuring that questions related to the accuracy or integrity of any part of the work are appropriately investigated and resolved. All procedures performed in this study involving human participants were in accordance with the Declaration of Helsinki (as revised in 2013). All participants gave informed consent and agreed to participate in the study. The study was approved by the Institute Research Ethics Committee of Affiliated Hospital of Nantong University (No. 2018-L100).

Open Access Statement: This is an Open Access article distributed in accordance with the Creative Commons Attribution-NonCommercial-NoDerivs 4.0 International License (CC BY-NC-ND 4.0), which permits the noncommercial replication and distribution of the article with the strict proviso that no changes or edits are made and the original work is properly cited (including links to both the formal publication through the relevant DOI and the license). See: https://creativecommons.org/licenses/by-nc-nd/4.0/.

\section{References}

1. Siegel RL, Miller KD, Jemal A. Cancer statistics, 2019. CA Cancer J Clin 2019;69:7-34.

2. Kuro-o M, Matsumura Y, Aizawa H, et al. Mutation of the mouse klotho gene leads to a syndrome resembling ageing. Nature 1997;390:45-51.

3. Ishii M, Yamaguchi Y, Yamamoto H, et al. Airspace enlargement with airway cell apoptosis in klotho mice: 
a model of aging lung. J Gerontol A Biol Sci Med Sci 2008;63:1289-98.

4. Kurosu H, Yamamoto M, Clark JD, et al. Suppression of aging in mice by the hormone Klotho. Science 2005;309:1829-33.

5. Goetz R, Nakada Y, Hu MC, et al. Isolated C-terminal tail of FGF23 alleviates hypophosphatemia by inhibiting FGF23-FGFR-Klotho complex formation. Proc Natl Acad Sci U S A 2010;107:407-12.

6. Hu MC, Shiizaki K, Kuro-o M, et al. Fibroblast growth factor 23 and Klotho: physiology and pathophysiology of an endocrine network of mineral metabolism. Annu Rev Physiol 2013;75:503-33.

7. Wolf I, Levanon-Cohen S, Bose S, et al. Klotho: a tumor suppressor and a modulator of the IGF-1 and FGF pathways in human breast cancer. Oncogene 2008;27:7094-105.

8. Lee J, Jeong DJ, Kim J, et al. The anti-aging gene KLOTHO is a novel target for epigenetic silencing in human cervical carcinoma. Mol Cancer 2010;9:109.

9. Abramovitz L, Rubinek T, Ligumsky H, et al. KL1 internal repeat mediates klotho tumor suppressor activities and inhibits bFGF and IGF-I signaling in pancreatic cancer. Clin Cancer Res 2011;17:4254-66.

10. Doi S, Zou Y, Togao O, et al. Klotho inhibits transforming growth factor-beta1 (TGF-beta1) signaling and suppresses renal fibrosis and cancer metastasis in mice. J Biol Chem 2011;286:8655-65.

11. Xie B, Zhou J, Yuan L, et al. Epigenetic silencing of Klotho expression correlates with poor prognosis of human hepatocellular carcinoma. Hum Pathol 2013;44:795-801.

12. Wang L, Wang X, Wang X, et al. Klotho is silenced through promoter hypermethylation in gastric cancer. Am J Cancer Res 2011;1:111-9.

13. Tang X, Fan Z, Wang Y, et al. Expression of klotho and $\beta$-catenin in esophageal squamous cell carcinoma, and their clinicopathological and prognostic significance. Dis Esophagus 2016;29:207-14.

14. Kuro-o M. Endocrine FGFs and Klothos: emerging concepts. Trends Endocrinol Metab 2008;19:239-45.

15. Wu X, Ge H, Gupte J, et al. Co-receptor requirements for fibroblast growth factor-19 signaling. J Biol Chem 2007;282:29069-72.

16. Ye X, Guo Y, Zhang Q, et al. $\beta$ Klotho suppresses tumor growth in hepatocellular carcinoma by regulating Akt/ GSK-3 $\beta$ /cyclin D1 signaling pathway. PLoS One 2013;8:e55615.

17. Lee KJ, Jang YO, Cha SK, et al. Expression of fibroblast growth factor 21 and $\beta$-klotho regulates hepatic fibrosis through the nuclear factor- $\kappa \mathrm{B}$ and c-Jun N-terminal kinase pathways. Gut Liver 2018;12:449-56.

18. Liu Z, Qi S, Zhao X, et al. Metformin inhibits $17 \beta$-estradiol-induced epithelial-to-mesenchymal transition via $\beta$ Klotho-related ERK1/2 signaling and AMPK $\alpha$ signaling in endometrial adenocarcinoma cells. Oncotarget 2016;7:21315-31.

19. Chang B, Kim J, Jeong D, et al. Klotho inhibits the capacity of cell migration and invasion in cervical cancer. Oncol Rep 2012;28:1022-8.

20. Lin BC, Wang M, Blackmore C, Desnoyers LR. Liverspecific activities of FGF19 require klotho beta. J Biol Chem 2007;282:27277-84.

21. Kurosu H, Choi M, Ogawa Y, et al. Tissue-specific expression of $\beta$ klotho and fibroblast growth factor (FGF) receptor isoforms determines metabolic activity of FGF19 and FGF21. J Biol Chem 2007;282:26687-95.

22. Motylewska E, Stępień T, Borkowska M, et al. Alteration in the serum concentrations of FGF19, FGFR4 and $\beta$ Klotho in patients with thyroid cancer. Cytokine 2018;105:32-6.

23. Poh W, Wong W, Ong H, et al. Klotho-beta overexpression as a novel target for suppressing proliferation and fibroblast growth factor receptor-4 signaling in hepatocellular carcinoma. Mol Cancer 2012;11:14.

24. Chen W, Zheng R, Baade PD, et al. Cancer statistics in China, 2015. CA Cancer J Clin 2016;66:115-32.

25. Yang J, Zhu J, Zhang YH, et al. Lung cancer in a rural area of China: rapid rise in incidence and poor improvement in survival. Asian Pac J Cancer Prev 2015;16:7295-302.

26. Zheng R, Zeng H, Zuo T, et al. Lung cancer incidence and mortality in China, 2011. Thorac Cancer 2016;7:94-9.

27. $\mathrm{Li} \mathrm{F,} \mathrm{Li} \mathrm{X,} \mathrm{Li} \mathrm{Z,} \mathrm{et} \mathrm{al.} \beta$ Klotho is identified as a target for theranostics in non-small cell lung cancer. Theranostics 2019;9:7474-89. Erratum in: Theranostics 2020;10:5528-9.

28. Nakai K, Yoneda K, Haba R, et al. $\beta$ Klotho expression is reduced in human non-melanoma skin cancer. Int J Dermatol 2015;54:e431-3.

29. Luo Y, Yang C, Lu W, et al. Metabolic regulator betaKlotho interacts with fibroblast growth factor receptor 4 (FGFR4) to induce apoptosis and inhibit tumor cell proliferation. J Biol Chem 2010;285:30069-78.

30. Liu Z, Zhang H, Ding S, et al. $\beta$ Klotho inhibits androgen/ androgen receptor-associated epithelial-mesenchymal transition in prostate cancer through inactivation of ERK1/2 signaling. Oncol Rep 2018;40:217-25.

31. Lin ZZ, Hsu C, Jeng YM, et al. Klotho-beta and 
fibroblast growth factor 19 expression correlates with early recurrence of resectable hepatocellular carcinoma. Liver Int 2019;39:1682-91. Erratum in: Liver Int 2020;40:2309.

32. Desnoyers LR, Pai R, Ferrando RE, et al. Targeting FGF19 inhibits tumor growth in colon cancer xenograft and FGF19 transgenic hepatocellular carcinoma models. Oncogene 2008;27:85-97.

33. Glass DJ. What's so special about FGF19-unique effects

Cite this article as: Zhou J, Ben S, Xu T, Xu L, Yao X. Serum $\beta$-klotho is a potential biomarker in the prediction of clinical outcomes among patients with NSCLC. J Thorac Dis 2021;13(5):3137-3150. doi: 10.21037/jtd-21-798 reported on skeletal muscle mass and function. Cell Metab 2017;26:287-288.

34. Kurosu H, Kuro-O M. The Klotho gene family as a regulator of endocrine fibroblast growth factors. Mol Cell Endocrinol 2009;299:72-8.

35. Lee S, Choi J, Mohanty J, et al. Structures of $\beta$-klotho reveal a 'zip code'-like mechanism for endocrine FGF signalling. Nature 2018;553:501-5. 